\title{
Physikalische Therapie: Therapiemittel Licht (2)
}

\author{
Jean-Michel Jeannin
}

Basel, Schweiz

\section{Einleitung}

Die vorliegende Folge befasst sich mit der Phototherapie von Dermatosen. Unter Phototherapie wird die Behandlung der Haut mit Licht von einer definierten Wellenlänge verstanden. Die biologischen Wirkungen des Lichts an der Haut werden hauptsächlich durch UV-Licht und nur in geringem Mass durch sichtbares Licht vermittelt (Abb. 1, 2) (Tab. 1) [1]. Aus der Fülle der publizierten Literatur zum Thema wurde eine arbiträre Auswahl getroffen, hauptsächlich nach dem Kriterium der Anschaulichkeit. Die Fülle des Stoffs macht ferner eine Aufteilung auf zwei Folgen notwendig.

\section{Wirkungsweise, Methoden und Anwendungsmöglichkeiten}

UV-Licht wird von der Haut entweder reflektiert oder absorbiert. UV-C-Licht $(<280 \mathrm{~nm})$ wird in der obersten Schicht der Haut, im Stratum corneum, absorbiert. UVA-Licht (320-400 nm) dringt tiefer in die Haut ein als UV-B-Licht (280-320 nm). Entsprechend wird UV-BLicht von Strukturen der Epidermis absorbiert, namentlich den Keratinocyten, Langerhans-Zellen und Melanocyten. Moleküle, die UV-Licht absorbieren, werden Chromophoren genannt. Wichtige Chromophoren der Haut sind Keratin, Melanin, Kollagen und Elastin sowie Urocaninsäure und die DNA [2]. Die Urocaninsäure weist photoprotektive Eigenschaften auf [3]. Unter UVLicht wird die trans-Urocaninsäure zur cis-Urocaninsäure umgewandelt [4]. Die Absorption von UV-Licht führt zu Änderungen der Struktur der absorbierenden Chromatophoren. Dergestalt veränderte Chromatophoren werden Photoprodukte genannt [2]. Die UV-Lichttherapie hemmt die Proliferation der Keratocyten, induziert die Apoptose von Immunocyten, hemmt Th1- und Th17Zellen und stimuliert Th2-Zellen [2]. UV-Licht stimuliert sowohl die Produktion von entzündungsfördernden (Prostaglandine) als auch jene von entzündungshem- menden Substanzen (unter anderem Interleukine). Schmalband-UV-Licht bewirkt eine Umstellung der Immunantwort von Th1 - zu Th2-Zellen [2]. Trotz aller Detailkenntnisse ist die Wirkungsweise noch nicht aufgeklärt.

Zur Ermittlung der notwendigen Dosis wird die minimale Erythem-Dosis bestimmt, d.h. die Dosis, die eine Rötung der Haut auslöst. In der Praxis hat sich jedoch durchgesetzt, die Dosis entsprechend dem Hauttyp des Patienten festzulegen (Hauttypen I-IV, wobei Hauttyp I der hellste Hauttyp ist und UV-Licht am wenigsten gut verträgt). Eine typische Dosis zur Behandlung der Psoriasis beträgt $0,2-0,6 \mathrm{~J} / \mathrm{cm}^{2}$ [5].

Sichtbares und infrarotes Licht wird im Rahmen der sogenannten Low-Level-Lasertherapie (LLLT) therapeutisch angewendet. Zurzeit verfügbare Daten zeichnen ein uneinheitliches Bild, was die postulierten Wirkungsmechanismen betrifft. Typische Wellenlängen des Lichts, das im Rahmen einer LLLT appliziert wird, sind z.B. 632,8, 670, 692, 780 und $786 \mathrm{~nm}$. Die LLLT wird eher den kom-

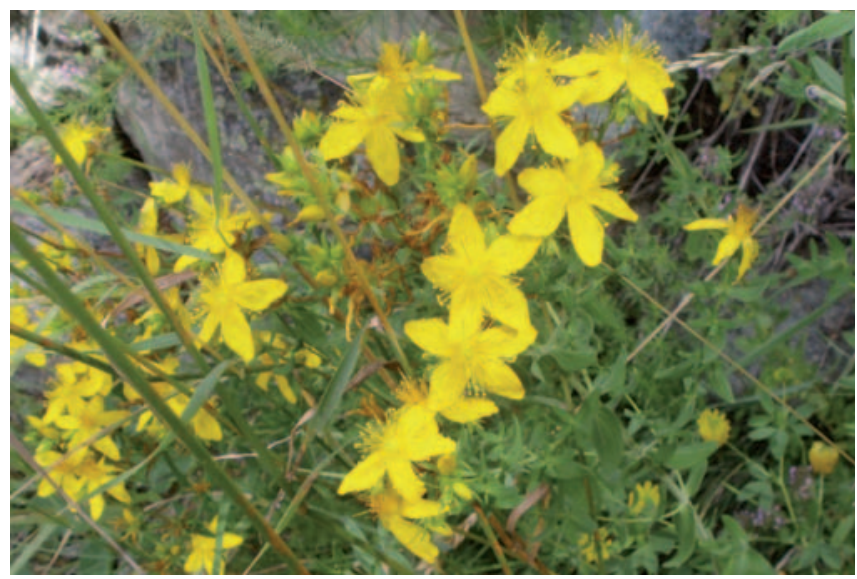

Abb. 1. Hypericum perforatum (Johanniskraut). Die Lichtblume Johanniskraut bietet eine wirksame Behandlung von Depressionen, die oft mit Lichtmangel vergesellschaftet sind. Die gleiche Pflanze kann bei dazu disponierten Personen zusammen mit UV-A-Licht eine polymorphe Lichtdermatose auslösen. Neuere Ergebnisse der Forschung lassen vermuten, dass Johanniskraut ein wirksames Mittel zur Behandlung von Hautkrankheiten sein könnte.

\section{KARGER}

() 2016 S. Karger GmbH, Freiburg

Fax +497614520714 Information@Karger.com www.karger.com 


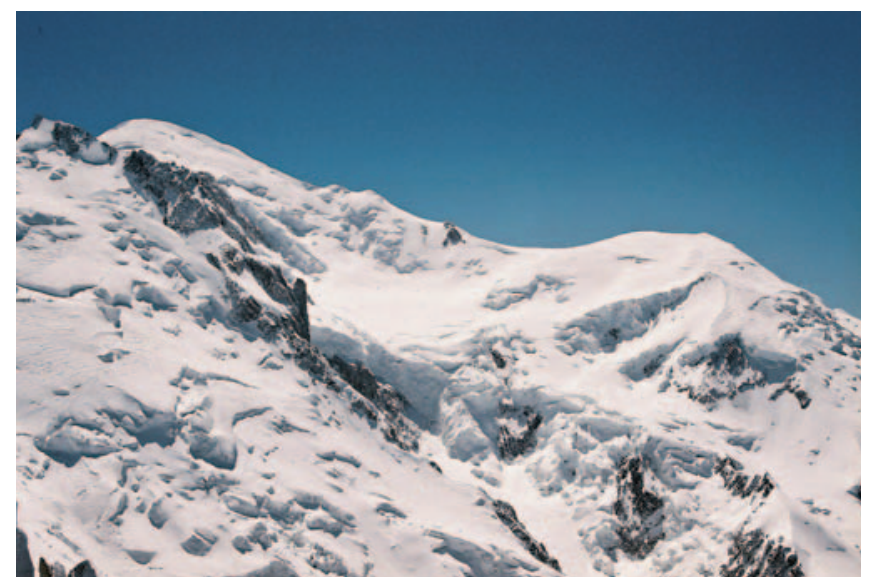

Abb. 2. Mont Blanc (4809 m), Frankreich. Die UV-Einstrahlung ist in den Hochalpen besonders intensiv, weil die Luft dünn und trocken ist. UVStrahlen, ob natürliche oder künstlich erzeugte, lösen in der Haut bestimmte Effekte aus, die sowohl schädigend als auch heilend wirken können. Mit UV-Lampen bestimmter Wellenlängen können einzelne dieser Effekte gezielt angesteuert werden.

plementärmedizinischen Methoden zugerechnet, was in Anbetracht der Bewertung der physikalischen Therapie durch die Schulmedizin logisch ist [6].

UV-A-Licht wird zur Behandlung des endogenen Ekzems («Neurodermitis») und von sklerosierenden Hautkrankheiten angewendet (Lichen sclerosus et atrophicus, Morphea, sklerodermiforme Graft-versus-Host-Erkrankung), UV-B-Licht zur Behandlung der Psoriasis, der Vitiligo, von Pruritus und des endogenen Ekzems ( «Neurodermitis») [1]. UV-A-Licht kann zusammen mit Psoralen verabreicht werden (PUVA-Therapie) [6]. Excimer-Laserlicht findet eine breite Anwendung bei der Behandlung der Psoriasis, der Vitiligo, des endogenen Ekzems sowie weiterer spezieller Dermatosen und Dermatitiden (Folliculitis, Alopecia areata, Leucoderm) [7]. Wundheilung und chronische Schmerzen sind die Domäne der LLLT [8].

\section{Klinische Erfahrung}

\section{Psoriasis}

In einer Metaanalyse von Almutawa et al. [9] wurde anhand von insgesamt 41 randomisierten placebokontrollierten klinischen Studien die Wirksamkeit von SchmalbandUV-B-Therapie, Schmalband-UV-B-Therapie kombiniert mit peroralen Medikamenten, Breitband-UV-B-Therapie, PUVA-Therapie und einer Kombination von PUVA-Therapie mit einem Bad in Salzwasser bewertet. Kriterien für den Behandlungserfolg waren entweder ein Psoriasis Area and Severity Index über 75 (PASI-75) oder Heilung. Ein PASI von 75 bedeutet eine Verminderung des PASI bei Eintritt um 75\% am Ende der Behandlung. In 9 Studien wurden 293 Patienten mit Schmalband-UV-B-Licht behandelt. Bei 62\% der Patienten besserte sich der PASI um
Tab. 1. Therapeutisch genutztes Licht [1]

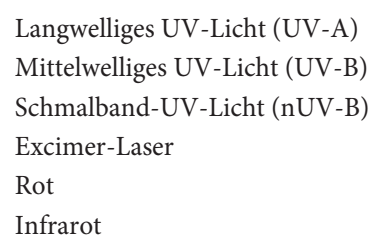

aBei Verwendung der gesamten Bandbreite von 280-320 nm spricht man auch von Breitband-UV-B (bUV-B).

mehr als 75\%. In einer Studie wurde die nUV-B-Lichtbehandlung mit der gleichzeitigen Verabreichung des Calcitriolderivats Calcipotriol kombiniert. 52\% erzielten eine Besserung des PASI von über 75\%. In einer anderen Studie wurde die nUV-B-Lichtbehandlung mit der gleichzeitigen Verabreichung des Retinoids Tazaroten kombiniert. 10\% der Patienten erzielten eine Verbesserung des PASI von mehr als $75 \%$. In 10 Studien waren $68 \%$ von 379 Patienten nach der Behandlung mit nUV-B-Licht frei von psoriatischen Hauterscheinungen. 90\% der Patienten waren frei von psoriatischen Veränderungen der Haut nach der Behandlung mit nUV-B und Calcitriol. Bei der BreitbandUV-B-Lichttherapie erzielten $73 \%$ von 246 Patienten aus 3 Studien eine Verbesserung des PASI um mehr als 75\%. Nur 39\% der Patienten erzielten eine solche Besserung, wenn sie vor der Phototherapie mit einem Bad in Salzwasser behandelt wurden. Im Unterschied dazu erreichten 76\% von 84 Patienten, die gleichzeitig mit einer Calcipotriolcrème behandelt wurden, eine Verbesserung des PASI um über 75\%. Mit einer Breitband-UV-B-Behandlung wurde in 4 Studien Symptomfreiheit für 59\% von 148 Patienten erzielt. Weder eine gleichzeitige Therapie mit Calcipotriolcrème bzw. -salbe, mit Teeröl noch mit dem topischen Glucocorticoid Fluocinonidcrème waren von Vorteil. Mit einer PUVA-Monotherapie erreichten $73 \%$ von 246 Patienten aus 8 Studien eine Besserung von über $75 \%$ des PASI. Mit einer PUVA-Monotherapie wurde in 10 Studien Symptomfreiheit für $79 \%$ von 372 Patienten erzielt. Eine zusätzliche Behandlung mit dem Retinoid Acitretin brachte in einer Studie für 94\% von 20 Patienten Symptomfreiheit. In einer anderen Studie erzielten mit der gleichen Kombination lediglich $63 \%$ von 30 Patienten eine Verbesserung des PASI von über $75 \%$. Die Behandlung mit Bädern und PUVA zeigte keine Vorteile gegenüber einer PUVA-Monotherapie. Bei 100\% von 34 Patienten wurde mit einer Kombination von PUVA, Bad und peroralen Retinoiden Symptomfreiheit erreicht. Die Autoren gelangten zu dem Schluss, dass von den Behandlungsmethoden mit UV-BLicht PUVA am besten wirksam ist, gefolgt von Schmalband-UV-B, Breitband-UV-B und PUVA kombiniert mit einer Badtherapie. Die Verträglichkeit war gut, und nur wenige Patienten schieden wegen unerwünschten Wirkungen aus. 
Eine begleitende stressmindernde Meditation kann die Abheilung von Psoriasis-Läsionen unter nUV-B-Behandlung deutlich beschleunigen [10]. Die Studie zeigt die Komplexität der Psoriasis eindrücklich. Sie veranschaulicht aber auch den Wert ganzheitlicher Behandlungen.

\section{Vitiligo}

Bei der Vitiligo handelt es sich um eine Depigmentierung der Haut. Ist das Gesicht betroffen, kann das zu schweren psychosozialen Einschränkungen führen. Eine Repigmentierung von über $75 \%$ erfuhren 3 Patienten mit einer ausgedehnten Vitiligo des Gesichts mit einer Kombination von $308 \mathrm{~nm}$ Excimer-Lasertherapie und lokaler
Verabreichung von Calcipotrien [11]. Der Erfolg einer Behandlung mit $308 \mathrm{~nm}$ Excimer-Laserlicht ist abhängig von der Lokalisation einer Vitiligo: Gesicht und Nacken sprechen sehr gut an - im Gegensatz zu Gelenkpartien und den Extremitäten [7].

\section{Anmerkung}

Die Phototherapie der atopischen Dermatitis, von Wunden und von Hautdegenerationen, die Aspekte der Verträglichkeit und Sicherheit sowie die Besprechung der Lichtdermatosen sind einer weiteren Folge vorbehalten.

\section{Literatur}

1 Armbruster MO, et al: 3.13 Phototherapie; in Gutenbrunner C, Glaesener JJ: Rehabilitation, Physikalische Medizin und Heilverfahren. Heidelberg, Springer, 2007, p 80.

$\checkmark 2$ Berneburg M, et al: Phototherapy with narrowband vs broadband UVB. Acta Derm Venereol 2005;85:98-108.

- 3 Barresi C, et al: Increased sensitivity of histidinemic mice to UVB radiation suggests a crucial role of endogenous urocanic acid in photoprotection. J Investig Dermatol 2011; 131:188-194.
4 Racz E, et al: Phototherapy and photochemotherapy for psoriasis. Dermatol Clin 2015;33: 79-89.

5 Lienhard A: Fototherapie der Psoriasis. Schweiz Z Dermatol 2015;17-20.

6 Chung H, et al: The nuts and bolts of low-level laser (light) therapy. Ann Biomed Eng 2012;40:516-533.

7 Mehraban S, Feily A: $308 \mathrm{~nm}$ excimer laser in dermatology. J Lasers Med Sci 2014;5:8-12.

8 Avci P, et al: Low-level laser (light) therapy (LLLT) in skin: stimulating, healing, restoring. Semin Cutan Med Surg 2013;32:41-52.
9 Almutawa F, et al: Systematic review of UVbased therapy for psoriasis. Am J Clin Dermatol 2013;14:87-109.

10 Kabat-Zinn J, et al: Influence of a mindfulness meditation-based stress reduction intervention on rates of skin clearing in patients with moderate to severe psoriasis undergoing phototherapy (UVB) and chemophototherapy (PUVA). Psychosom Med 1998;60:625632.

11 Mouzakis JA, et al: Rapid response of facial vitiligo to $308 \mathrm{~nm}$ excimer laser and topical calcipotriene. J Clin Aesthet Dermatol 2011; 4:41-44. 